\title{
Miniaturized Dual-band RFID Antenna Applied for Smart Home
}

\author{
Yang Guohui* ${ }^{1}$, Wang Erchao ${ }^{1}$, Tang Henghe ${ }^{2}$, Ma shuang ${ }^{1}$ and Gu Xuemai \\ ${ }^{1}$ School of Electronics and Information Engineering, Harbin Institute of Technology, \\ Harbin, 150001, China \\ ${ }^{2}$ Chinese Academy of Sciences, Beijing, 100000, China \\ gh.yang@hit.edu.cn
}

\begin{abstract}
As one of the most important components in wireless communication system, antenna's size is a limit to its further development. Two kinds of novel Radio Frequency Identification (RFID) antenna is proposed, which is base on conventional dipole antenna. Through arms carried by bending or simply fractal, its size and electrical length can be decreased compared with the same frequency of the antenna. These two kinds of symmetric antennas can realize miniaturization and dual band. Because of its small size, it could be employed by smart home. And we make change and optimization to symmetric model of the existing antennas through a large number of simulation analyses. The experiment on the processing objects shows the antennas have a good performance.
\end{abstract}

Keywords: RFID; Smart Home; Antenna; miniaturization; dipole; dual band

\section{Introduction}

Smart home is a network which is used to connect any item in the house with the Internet by sensor device according to the agreed protocol. It can exchange information and communicate in order to realize intelligent identification location, tracking, monitoring and management. The kernel theory of the smart home is to make any object connect to the Internet and realize intellectualization. RFID is a kind of technology which could make the item be able to "speak". RFID is a much advanced technology of non-contact automatic identification at present, and its basic principle is using the radio frequency signal and the space coupling transmission characteristics to realize the object of the automatic identification. Based on the frequency, RFID applications could be divided into lowfrequency (LF), high Frequency (HF), ultra high frequency (UHF), and microwave (MW). The representations of the corresponding frequencies are as follows: below $135 \mathrm{KHz}$ at LF, $13.56 \mathrm{MHz}$ at $\mathrm{HF}, 860 \mathrm{M}-960 \mathrm{MHz}$ at $\mathrm{UHF}$ and $2.4 \mathrm{GHz}, 5.8 \mathrm{GHz}$ at $\mathrm{MW}$. To reduce the costs fully, and conserve the equipment's, and operate in the smart home easily, it is necessary to improve the antenna in RFID. It needs to normalize the model of antennas to realize the multi-purpose property, in order to make the antenna work in multiple bands with adequate screening, and realize multi-frequency radio frequency identification.

In recent years, wireless communication technologies such as radio frequency identification (RFID) and mobile communications have rapidly developed. As one of the most important components in wireless communication system, antenna's size is a limit to its further development. So miniaturized antenna design plays an important role in wireless communication system miniaturization, high integration, and high performance. For RFID systems, antenna for receiving electromagnetic radiation is mainly divided into two categories: read head antenna and tag antenna. Tag antennas are mostly printed dipole 
antennas generally in the form of deformation structures. Some applications also require the tag antenna have circular polarization characteristics. In the existing research, there are many areas to improve: the traditional PIFA antenna requires a large floor to get to their excellent performance, which does not match with the current requirements of small mobile terminal; built-in monopole can achieve multi-frequency and broadband, but its volume is too large compared with the floor, which does not meet the current demand for miniaturization; circular monopole antenna combinations can cover multiple bands, but its frequency bandwidth is too narrow and difficult to regulate.

Dipole antenna is one of the most common forms of antennas, because of its simple structure and low cost. And it has been widely used in a variety of communications and radar systems. This paper is to design a different arm rounded U-shaped antenna and round notched antenna. The two kinds of symmetric antennas can realize miniaturization and dual band. And we make change and optimization on the symmetric model of the existing antenna through a large number of simulation analyses. The experiment shows the antenna have a good performance.

\section{Antennas Theory and Design}

Conventional dipole antenna is shown in Figure 1. Symmetrical dipole is a symmetrical center fed dipole, and its arms are constituted by two conductive wires of equal length. The radius of the arm wire is $a$, and length is $l$, the total length is $2 l$. Usually used antenna is the half-wave antenna, which $2 l=\lambda / 2$.And also current on the arm is symmetrical.

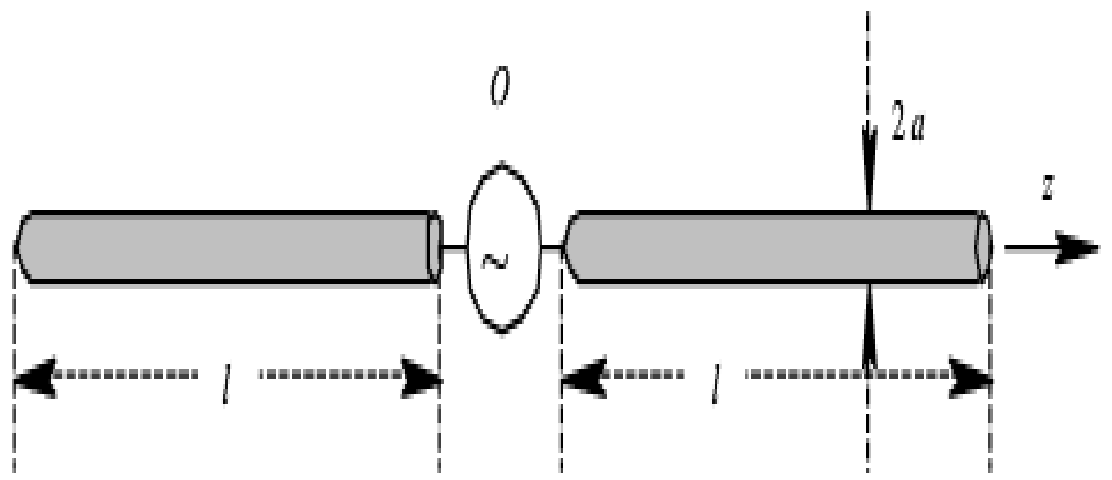

Figure 1. Basic Structure of the Conventional Dipole Antenna

Operating frequency of the antenna is determined by the electrical length of the antenna, and the antennas' characteristics relate to their shape. For the U-shaped dipole antenna, whose arms carried by bending or simply fractal, its electrical length size can be decreased compared by the same frequency of the antenna. So it can achieve miniaturization of the antenna through this method. In this paper, an antenna is improved the external shape, mainly the arms of the U-type and proportion of the length to be adjusted. And to realize dual band of the antenna, this paper employs unequal arms of the U-shaped dipole.

Meanwhile, the dipole antenna can be fed in a variety of ways. This article, we have adopted a center-fed coaxial mode, and it also adds more consideration when the antenna designs. To solve the problem of feed, the antenna will be distributed symmetrically arms on both sides of the substrate, and Align the center of feed zone on each side in a line. Coaxial probe is connected with the arm on top layer, and the coaxial outer conductor is connected 
with the arm on bottom layer. So thought this method it can minimize the effect of the antenna shape parameters, and have little effect to the operating characteristics of the antenna.

\section{Simulation and Optimization}

\subsection{U-shaped Dipole Antenna with Different Arm Length}

The physical model is shown in Figure 2(a) as follows: based on the half-wave dipole, we increase a pair of harmonic different length arm to realize a U-shaped dipole antenna. The substrate of the antenna is FR-4, and it is covered double-sided copper with dielectric constant of 4.9, dielectric substrate thickness $1.4 \mathrm{~mm}$, Copper thickness $0.2 \mathrm{~mm}$. Two symmetrical U-shaped patch place at each layer of the substrate.

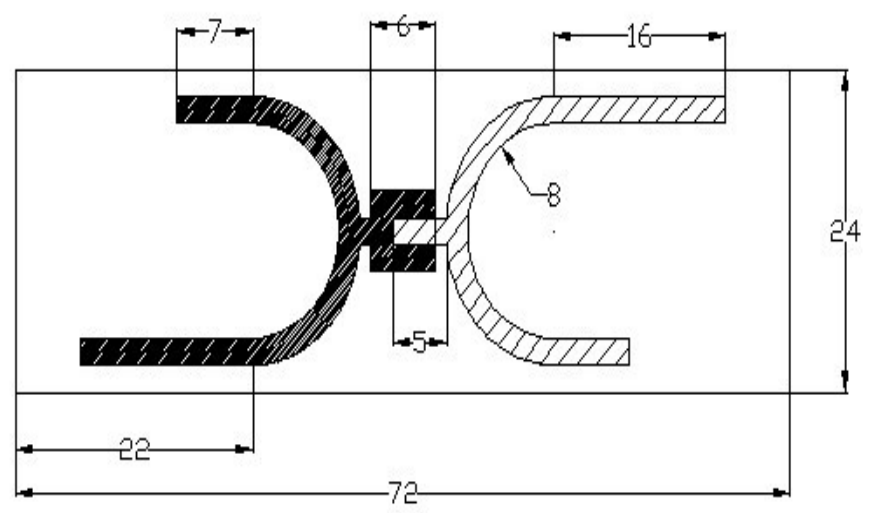

(a)

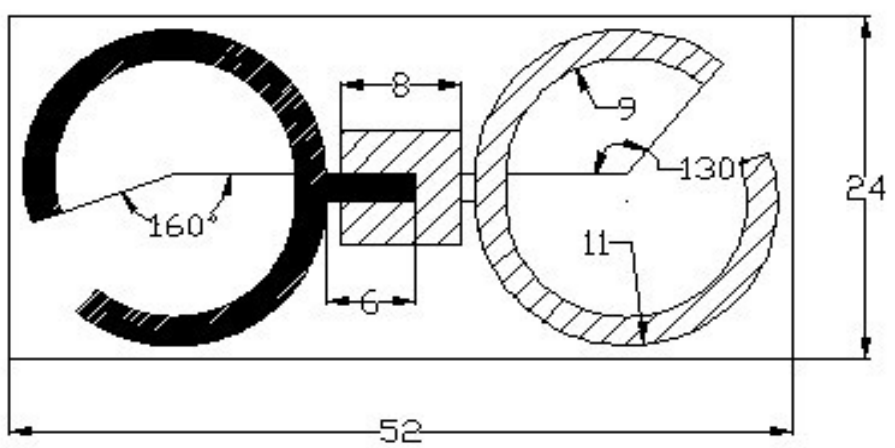

(b)

Figure 2. Two Kinds of Novel Radio Frequency Identification (RFID) Antenna Proposed (a) U-shaped Dipole Antenna. (b) Round Notched Antenna

In Simulation, parameters optimization is a large work. We can change the shape of the antenna on several aspects: 1 . the radius R; 2 . the different length of the arms L1, L2; 3. the feed zone parameters M1, M2. These factors will get antenna frequency distribution and varying degrees of impact. By adjusting these parameters, we can get to obtain maximum impedance bandwidth, and frequency response.

Through a large number of simulation calculations and adjustments by using CST, the final optimization parameters can select as the following dimensions: R1 $=8 \mathrm{~mm}, \mathrm{~L} 1=7 \mathrm{~mm}, \mathrm{~L} 2=$ $16 \mathrm{~mm}, \mathrm{M} 1=5 \mathrm{~mm}, \mathrm{M} 2=6 \mathrm{~mm}$. Size of the substrate:X1 $=72 \mathrm{~mm}, \mathrm{Y} 1=24 \mathrm{~mm}$. Because the 
antenna has many parameters, and bandwidth is affected by many parameters, so in the simulation and optimization process, firstly fix some part of the parameters, then the calculated value preferably gradually adjust several parameters fixed, the optimal frequency response simulation results can be seen in Figure 3.

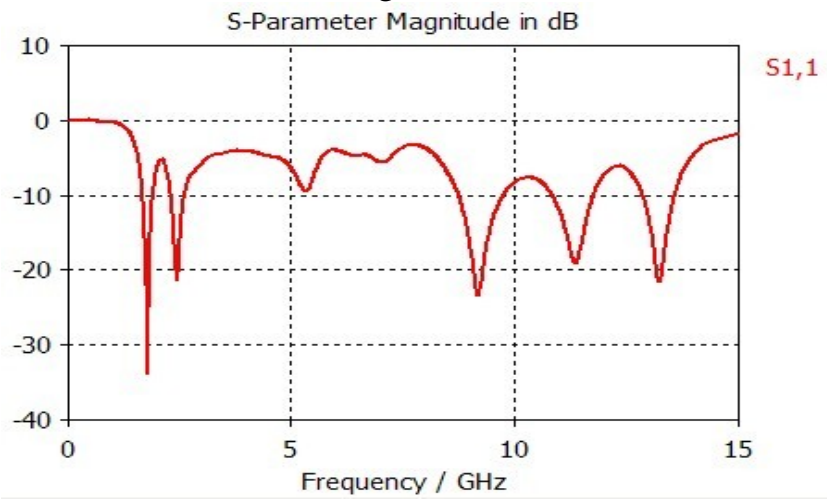

Figure 3. The Frequency Response Simulation of the U-shaped Dipole Antenna

\subsection{Round Notched Dipole}

Through the simulation results of different arm length of the U-shaped antenna, its size is larger, which not quite satisfy the requirements of small antennas. Therefore, the shape of the antenna has been improved again. The arms are bent to form a round notched dipole based on the U-shaped antenna model with different length of the arm.

In this antenna model, adjust by changing the parameters to simplify the model. The parameters are considered into the radius of circular time around R2, the gap position angle and the notch angle and so on. Through a large number of simulation calculations and adjustments by using CST, the final optimization parameters can select as the following dimensions in Figure 2(b). Through simulation calculated, the optimal antenna model simulation result is as Figure 4.

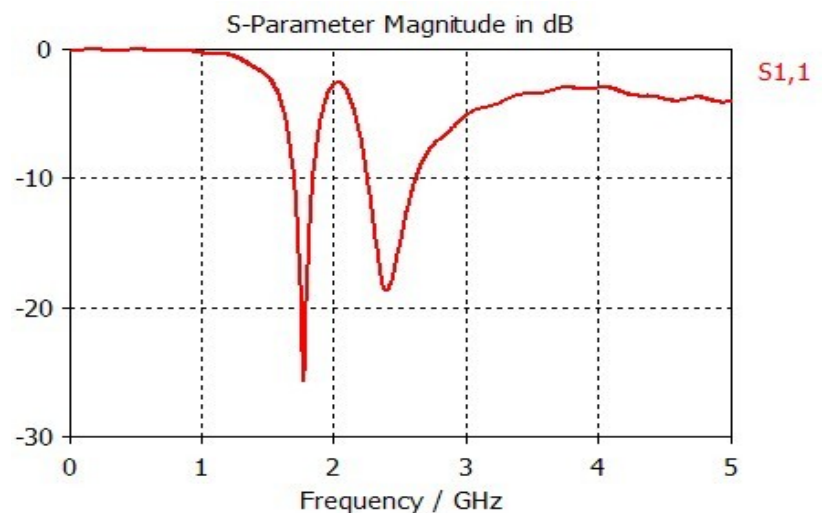

Figure 4. The Frequency Response Simulation of the Round Notched Antenna

\section{Experiment and Results}

By using CST simulation, debugging and improvement on continuous formation of the optimal model, it was processed physical and taken measurements in a microwave chamber to test the antenna actual performance. And at last we compare the results of the two kinds of antenna. 


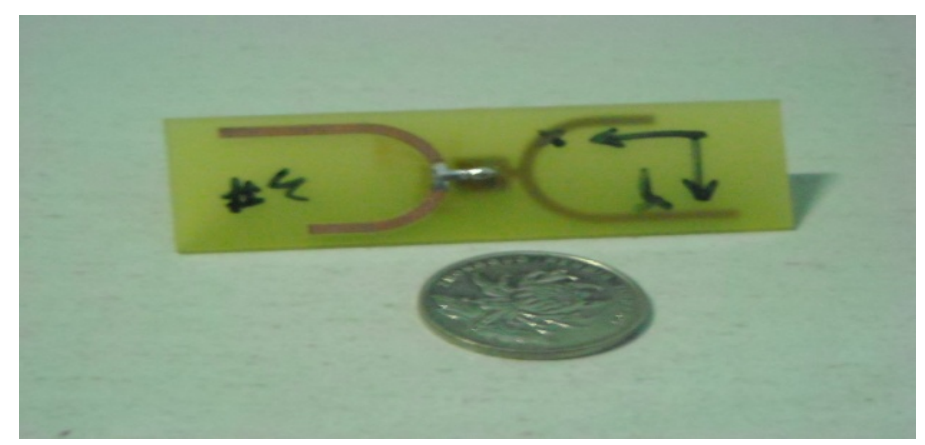

Figure 5. The Processed Physical of the U-shaped Dipole Antenna

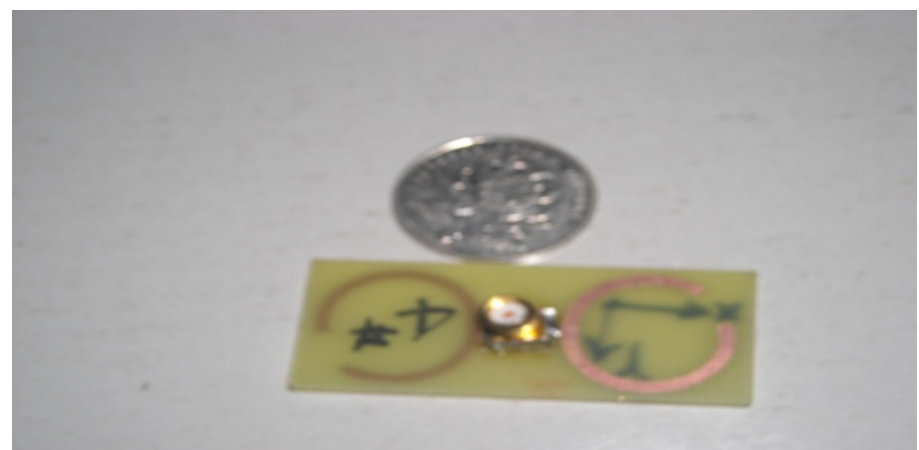

Figure 6. The Processed Physical of the U-shaped Dipole Antenna

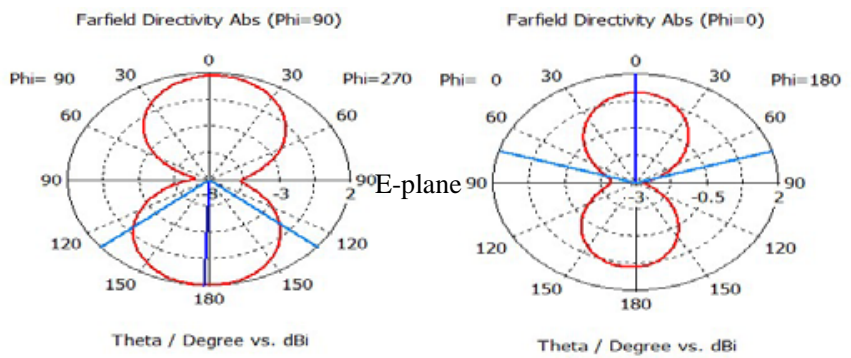

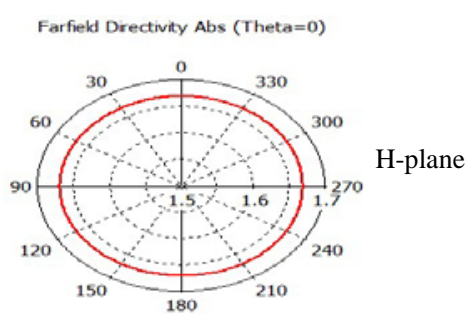

Phi / Degree vs. dBi

a

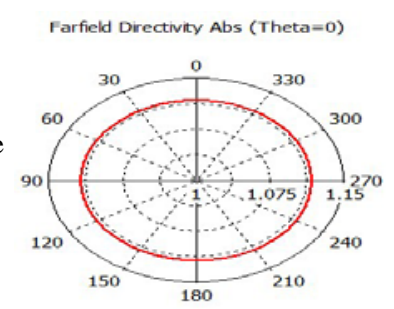

Phi / Degree vs. dBi

$\mathrm{b}$

Figure 7. Simulation Result of the Far-field pattern (a) U-shaped Dipole Antenna about its Far-field pattern at $1.80 \mathrm{GHz}$ on E-plane and $\mathrm{H}$-plane (b) The Round Notched Dipole about its Far-field Pattern at $1.78 \mathrm{GHz}$ on E-plane and H-plane 
The processed physical of two kinds of antenna are shown in Figure 5 and Figure 6. Through measurements, the electrical length of the U-shaped dipole antenna is $0.425 \lambda$, and the electrical length of the round notched dipole is $0.308 \lambda$. So we can see whose arms carried by bending or simply fractal, the antenna's electrical length size can be decreased compared by the same frequency of the antenna. It shows the round notched dipole can achieve miniaturization better, and its far-field pattern is better than the U-shaped dipole antenna at a low frequency.

We can see the round notched dipole has dual band center frequency at $1.78 \mathrm{GHz}$ and2.40GHz. At $1.78 \mathrm{GHz}$, Its bandwidth is from $1.70 \mathrm{GHZ}$ to $1.85 \mathrm{GHZ}$, and its absolute bandwidth is $8.4 \%$. Its gain is $1.812 \mathrm{~dB}$ in figure 7 . At $2.40 \mathrm{GHz}$, Its bandwidth is from $2.25 \mathrm{GHZ}$ to $2.62 \mathrm{GHZ}$, and its absolute bandwidth is $15.4 \%$. Its gain is $2.07 \mathrm{~dB}$.

And we can get the U-shaped dipole antenna has dual band center frequency at $1.80 \mathrm{GHz}$ and $2.45 \mathrm{GHz}$. At $1.80 \mathrm{GHz}$, Its bandwidth is from $1.72 \mathrm{GHz}$ to $1.91 \mathrm{GHz}$, and its absolute bandwidth is $10.56 \%$. Its gain is $2.316 \mathrm{~dB}$, and its far-field pattern is shown in figure 7 . At $2.45 \mathrm{GHz}$, Its bandwidth is from $2.30 \mathrm{GHz}$ to $2.61 \mathrm{GHz}$, and its absolute bandwidth is $12.65 \%$. Its gain is $2.01 \mathrm{~dB}$.

\section{Conclusions}

This article is based on the traditional symmetric dipole antenna. And through bold innovative improvement, and overcoming the difficulties of shape and feed applications, we change the structure and size of the antenna. Through extensive simulation and calculation, the two optimized dipole antennas have been gained, and the corresponding simulation results and experimental data have been given. They both have good working performance at frequency and gain, the basic realization of multi-band and miniaturization. To realize dual band of the antenna, this paper employs different unequal arms of the U-shaped dipole. And to realize miniaturization, we make arms carried by bending or simply fractal. It is foreseeable that this form of printed symmetric dipole can meet the corresponding application requirements by rational design. And experiments show the two antennas have a good performance and the round notched dipole can achieve miniaturization better in miniaturization.

\section{Acknowledgment}

The author thanks for the assist of Prof.Wu Qun ,Dr.Yang Guo Hui and classmate Tang Henghe, Liu Xiaoxin. And thanks for the support of Science and Technology on Communication Information Security Control Laboratory. Sponsored by the National Natural Science Foundation of China under Grant 60801015 and 61001036, the special funds projects for technological and innovative talent of Harbin City (2010RFXXG010), the Fundamental Research Funds for the Central Universities (No. HIT. IBRSEM. 2009), the Fundamental Research Funds for the Central Universities (No. HIT. NSRIF. 201153), China Postdoctoral Science Foundation funded project (No. LBHZ10126), Heilongjiang Postdoctoral Foundation project (No. 20110491093).

\section{References}

[1] S. Honda, M. Ito, H. Seki and Y. Jinbo, “A disc monopole antenna with 1:8 impedance bandwidth and omnidirectional radiation pattern”, Proc ISAP92,Sapporo, Japan, (1992), pp. 1145-1148.

[2] J. W. Wang and Z. B. Kuang, "The interaction between crack and electric dipole of piezoelectricity”, Acta Mech. Solida Sin, (2000). 
[3] Z. M. Xiao and B. J. Chen, "A screw dislocation interacting with a coated fiber", Mechanics of Materials, 2000 E. Taillefer, Direction-of-arrival estimation using radiation.

[4] A. Edalati and T. A. Denidni, "High-Gain Reconfigurable Sectoral Antenna Using an Active Cylindrical FSS Structure”, Antennas and Propagation, IEEE Transactions on, vol. 59, (2011), pp. 2464-2472.

[5] C. H. Cao, Y. P. Ding and X. G. Yang, "A 24-GHz transmitter with on-chip dipole antenna in $0.13 \mu \mathrm{m}$ CMOS”, IEEE J Solid-State Circuits, (2008).

[6] K. C. L. Chan, X. J. Tian and Y. Huang, "Integrated antenna for future wireless communication applications”, Proc 2rd European Conf An- tennas and Propagation, (2007).

[7] J. J. Lin, G. Li, A. Sugavanam, G. Xiaoling, L. Ran, J. E. Brewer and K. K. O., "Integrated antennas on silicon substrates for communication over free space”, Electron Device Letters IEEE, (2004).

[8] K. Kimoto, N. Sasaki and M. Nitta, "Inter-chip transmission characteristics of SI on-chip integrated antennas”, International Symposium on Antennas and Propagation Society, (2007). 
International Journal of Smart Home Vol.7, No.5 (2013) 